\title{
Eivolução do programa de controle reprodutivo de cães e gatos realizado na Unesp, Campus de Jaboticabal-SP, no período de 2007 a 2014
}

\section{Evolution of reproductive control program for dogs and cats, held at Unesp, Jaboticabal-SP, from 2007 to 2014}

\section{Resumo}

O controle da população de cães e gatos é uma preocupação para a saúde pública. Este relato descreve as atividades realizadas no Projeto de Extensão da Faculdade de Ciências Agrárias e Veterinárias da Universidade Estadual Paulista "Júlio de Mesquita Filho" (UNESP) de Jaboticabal, localizada no Estado de São Paulo, Brasil, que visou à esterilização cirúrgica de cães e gatos, com ações sociais aliadas ao ensino e pesquisa. Em 2007, ano de implantação do projeto, foram esterilizados 129 animais; já no ano de 2014, entre os meses de janeiro e outubro, foram esterilizados 1.538 animais, o que demonstra a evolução e aceitação do programa pela sociedade local. Toda a atividade realizada colaborou para a formação acadêmica e profissional dos alunos do projeto de extensão, bem como residentes participantes do programa. Pode-se concluir que o desenvolvimento de programas para a esterilização de cães e gatos, em conjunto com instituições educacionais, aliando pesquisa às práticas de ensino e extensão, é uma opção viável para as práticas de ensino em Medicina Veterinária.

\section{Summary}

The population control of dogs and cats is a public health concern. This report describes the activities of the Academic Extension of the College of Agricultural and Veterinary Sciences of UNESP in Jaboticabal/SP - Brazil aimed at surgical sterilization of stray dogs and cats, with social actions related to teaching and research. In 2007, the first year of the project, 129 pets were sterilized, whereas in 2014, between January and October, 1538 animals were sterilized, which shows the increase in program acceptance by the local community. All the activities carried out contributed towards the academic and professional training of students of the extension project, as well as residents participating in the program. It can be concluded that the development of surgical sterilization programs of dogs and cats by educational institutions, combining research, teaching, and extension practices, is a viable option for veterinary medicine teaching practices. 
B. I. Macente ${ }^{1}$

A. Tartarelli ${ }^{1}$

L. A. Lins ${ }^{1}$

L. M. Leal' ${ }^{1}$

T. C. Prada ${ }^{1}$

C. M. J. Miranda ${ }^{1}$

C. E. P. Belo ${ }^{1}$

I. C. Canavari'

L. W. Campos'

M. V. Montans ${ }^{2}$

T. B. Corsini ${ }^{1}$

R. V. P. Oliveira ${ }^{1}$

M. Apparício'

P. Tosta ${ }^{1}$

G. H. Toniollo'

J. F. Lui'
Via de Acesso Prof. Paulo Donato Castellane, s/n., 14884-900,

Jaboticabal-SP, Brasil.

更 beatrice.vetuel@yahoo.com.br

\section{Palavras-chave}

Caninos. Felinos. Universidades. Programa.

Esterilização cirúrgica.

\section{Keywords}

Canines. Felines. Universities. Program. Surgical sterilization.
1 Universidade Estadual Paulista - UNESP, Campus Jaboticabal/SP

2 Centro Universitário Barão de Mauá, Ribeirão Preto/SP

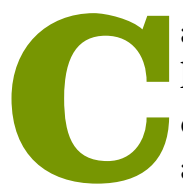

ães e gatos convivem com os seres humanos há milênios. Essa interação leva à formação de um círculo de relações entre as saúdes humana e animal e o meio ambiente (BEAVER, 2001). Quando esse círculo está em equilíbrio, a convivência entre seres humanos e animais gera benefícios psicológicos, fisiológicos e sociais. No entanto, a criação de cães e gatos sem cuidados veterinários, mobilidade irrestrita e sem controle reprodutivo determina alterações nos padrões de bem-estar dos animais que podem resultar na ocorrência de agravos aos seres humanos, como agressões, transmissão de doenças e contaminação ambiental (ARMSTRONG; BOTZLER, 2008).

A possibilidade de ocorrerem transtornos nas relações estabelecidas entre os animais de companhia e os seres humanos está diretamente relacionada às condições sociais, econômicas e culturais da população (VIEIRA et al., 2009). Em algumas cidades brasileiras, a relação entre cães e pessoas chega a 1:3, como em Curitiba e Piraquara, no Paraná (BIONDO et al., 2007; MARTINS et al., 2013), e a de gatos representa $25 \%$ da estimativa canina (ALVES et al., 2005). Em alguns países da Europa, a população de gatos já ultrapassou a de cães (MARCHAND; MOORE, 1991), o que em breve também será realidade no Brasil (GENARO, 2010).

Existe um despreparo de grande parte da população detentora de animais de companhia sobre como desenvolver a posse ou a sua guarda responsável. Esse fato, associado ao desconhecimento do comportamento reprodutivo, idade de amadurecimento sexual, número de descendentes e medidas adequadas de manejo nutricional e higiênico-sanitário, colabora para a ocorrência de abandono e aumento 
expressivo de animais sem controle (BEAVER, 2001; THRUSFIELD, 2004). A Organização Mundial da Saúde (2000) listou como os principais problemas provocados pelo excesso de animais sem controle de mobilidade e supervisão: a transmissão de doenças, principalmente zoonoses (raiva, leishmaniose e toxoplasmose), a proliferação de parasitas (pulgas, carrapatos e sarna), agressões (arranhões e mordeduras), acidentes de trânsito, poluição por dejetos, poluição sonora, entre outras perturbações.

Medidas devem ser tomadas para controlar os desequilíbrios nas populações de animais de companhia. É necessário que, juntamente com a participação ativa da sociedade, o poder público elabore políticas que visem à melhoria da situação dos animais sem controle (ACHA; SZYFRES, 2003). No Estado de São Paulo, os animais encontrados nas ruas sem identificação eram recolhidos e eutanasiados pelos centros de controles de zoonoses como forma de controle populacional e de zoonoses de relevância, tais como a raiva e a leishmaniose. No entanto, essa prática apresentava altos custos de realização e se contrapunha a preceitos sociais e éticos (ALMEIDA et al., 2014; SANTANA; OLIVEIRA, 2008). Em 16 de abril de 2008, foi promulgada a Lei Estadual número 12.916 (SÃO PAULO, 2008), que dispôs sobre a proibição da eutanásia em animais saudáveis e o controle da reprodução de cães e gatos.

A Organização Mundial para Saúde Animal (OFFICE INTERNATIONAL DES EPIZOOTIES, 2010) indicou como estratégia satisfatória de controle populacional de animais domésticos a educação da população para guarda responsável, legislação específica, registro geral e identificação do animal, associada ao controle reprodutivo por métodos cirúrgicos e não cirúrgicos, recolhimento e manejo de cães de rua, controle das fontes de alimento e abrigo, restrição do movimento e educação para a redução dos casos de mordeduras e eutanásia. O Brasil não possui uma política nacional de manejo populacional de cães e gatos, e essa ação tem sido realizada de forma isolada em alguns Estados e municípios (GARCIA; MALDOMADO; LOMBARDI, 2008), como as cidades de São José dos Pinhais/PR (CATAPAN et al., 2014) e Rio de Janeiro/RJ (JOFFILY et al., 2013).

A esterilização de cães e gatos, quando realizada em animais saudáveis, é considerada um procedimento seguro e com baixa morbidade e mortalidade, sendo eficiente para o controle populacional, principalmente em fêmeas (CONCANNON; MEYERS-WALLEN, 1991; MACKAY, 1993). Entretanto, um programa de esterilização pode ser mais efetivo, quando houver a interação de vários setores da sociedade (ORGANIZAÇÃO PAN-AMERICANA DE SAÚDE, 1999), principalmente da junção de poderes públicos com instituições universitárias, por meio de projetos de extensão (MATERA, 2008).
As atividades de extensão realizadas pelas universidades promovem um processo educacional, cultural e científico que permite a expansão e a divulgação de conhecimentos adquiridos pelos alunos nas salas de aula por meio de atividades ligadas à sociedade (SILVA; VASCONCELOS, 2006).

\section{Objetivo}

Este trabalho teve por objetivo a descrição das atividades realizadas do Projeto de Extensão da Faculdade de Ciências Agrárias e Veterinárias da Universidade Estadual Paulista "Júlio de Mesquita Filho" (UNESP) de Jaboticabal, intitulado "Levantamento sérico de leptospirose e erliquiose em fêmeas caninas de proprietários de baixa renda encaminhadas para a esterilização em Jaboticabal-SP", que visou à esterilização cirúrgica de cães e gatos, desenvolvida com a participação de alunos em atividade de extensão, residentes, veterinários pós-graduandos pesquisadores, professores e voluntários.

\section{Descrição do projeto}

Os recursos financeiros foram obtidos por meio de convênios firmados com a Prefeitura Municipal, a Associação Protetora dos Animais de Jaboticabal (APA), a Pró-Reitoria de Extensão Universitária (PROEX)/UNESP e a Fundação de Apoio à Pesquisa, Ensino e Extensão (FUNEP). As cirurgias foram realizadas duas vezes por semana, às terças e quartas-feiras, sempre no período da manhã no Centro de Esterilização de Cães e Gatos (CECG), no campus da universidade. $\mathrm{O}$ projeto contou com a participação de voluntários externos e internos à universidade, alunos bolsistas do projeto de extensão, alunos da graduação do curso de Medicina Veterinária e estagiários da universidade, submetidos à orientação de médicos-veterinários residentes (seis pessoas), pós-graduandos, pesquisadores e professores vinculados à universidade, com uma participação média de 25 pessoas.

Os proprietários interessados em incluir o animal para castração no programa, bem como os voluntários e a Prefeitura, ao recolherem animais não domiciliados, entravam em contato com o CECG e realizavam um pré-cadastro por telefone ou pessoalmente, agendando um dia para a possível realização da cirurgia. As informações referentes aos animais, obtidas nos formulários de cadastramento, incluíam espécie, sexo, idade, estado sanitário e localidade. Nessa data, os cães e gatos eram submetidos à avaliação clínica pelos residentes e alunos, e os que apresentaram condições de higidez foram encaminhados para cirurgia. O responsável pelo animal preenchia um formulário, com todos os dados pessoais e referentes ao animal, assentindo na realização do procedimento cirúrgico. 
Os alunos bolsistas ficavam responsáveis pelo preparo, organização e esterilização de todos os materiais cirúrgicos empregados na castração, como confecção dos fios cirúrgicos, reposição dos medicamentos, entre outros - sendo fundamentais para o bom andamento do programa.

As cirurgias foram realizadas mediante aplicação de medicações pré-anestésicas, seguidas de anestesia geral injetável (tiletamina e zolazepan, $0,2 \mathrm{~mL} / \mathrm{Kg}$, aplicados por via intramuscular). As técnicas cirúrgicas empregadas foram ovariectomia para fêmeas caninas e felinas pré-puberes e ovario-histerectomia para as púberes. Essa diferenciação para com as fêmeas pré-puberes foi adotada por não ser necessária a realização de histerectomia em um animal que ainda não recebeu estímulos hormonais sobre os tecidos uterinos, a não ser que houvesse uma indicação específica para sua remoção (GOETHEM; OKKENS; KIRPENSTEIJN, 2006). Destaque-se ainda que a ovariectomia é um procedimento pouco invasivo, de menor tempo operatório e baixa morbidade (ROMAGNOLI, 2008). Nos machos, foram empregadas as técnicas de castração pré-escrotal para cães e escrotal para gatos. Todas as fêmeas, caninas e felinas, e os gatos machos foram tatuados com um " $\mathrm{C}$ " na parte interna da orelha com o intuito de facilitar a identificação futura do animal já castrado.

Os tutores dos animais foram informados pelos alunos sobre as medidas necessárias para o estabelecimento da guarda responsável, explicando os cuidados necessários como vacinações, controle de endo e ectoparasitas, instruções nutricionais, disponibilidade de água potável, cuidados de higienização (animal e ambiente) e os riscos de doenças zoonóticas. Além dessa orientação, ao final dos atendimentos foram fornecidos folders confeccionados pela APA com as mesmas orientações detalhadas.

Em paralelo aos procedimentos de castrações, também foram coletados dados e/ou amostras de sangue, urina e material dispensado durante as cirurgias, tais como testículos e ovários, para serem utilizados em projetos de pesquisa desenvolvidos na pós-graduação da universidade.

\section{Resultados e Discussão}

No início do projeto, em julho de 2007 , foram atendidos 129 animais de ambas as espécies, sendo 88 caninos e 41 felinos. As fêmeas representaram $81 \%$ das esterilizações. Destas, 64 (72\%) foram cadelas e as demais gatas. A média em seis meses foi de 21,5 esterilizações por mês. No segundo ano do projeto, 600 animais foram esterilizados, sendo que 426 (71\%) eram fêmeas de ambas as espécies. Destas, as fêmeas caninas representaram 63\%. Houve um incremento de 471 animais (465\%) em relação ao ano anterior e a média mensal alcançou a marca de 50 esterilizações. Já em 2009, foram esterilizados 985 animais, ou seja, 385 a mais que em 2008 (164\%), com média de 82 animais esterilizados por mês (Figura 1).

\section{Animais Castrados}

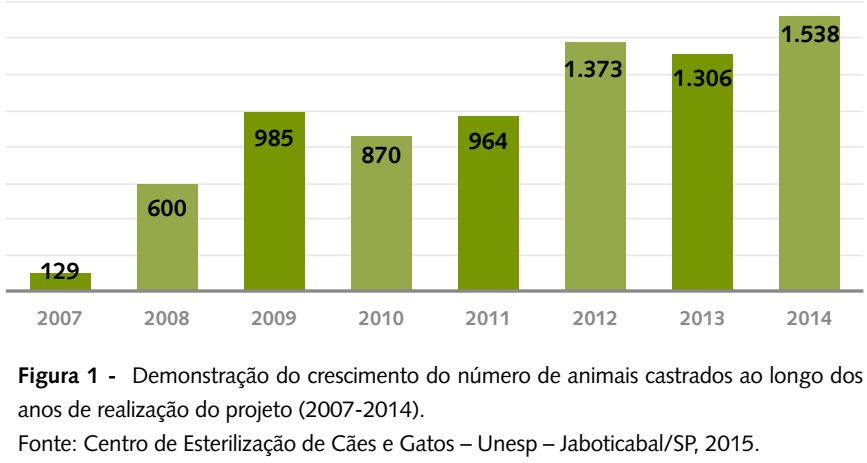

A observação da Figura 1 revela que no transcurso do projeto houve o crescimento constante e permanente do número de animais castrados. A análise comparativa dos dois extremos (2007 e 2014) revela um incremento de cerca de 12 vezes.

No ano de 2014, entre os meses de janeiro e outubro, foram esterilizados 1.538 animais. Destes, 767 (49,87\%) eram caninos, dos quais 151 (9,82\%) machos e 616 $(40,05 \%)$ fêmeas. Para a espécie felina, foram castrados $771(50,13 \%)$ animais, sendo $232(15,08 \%)$ machos e 539 $(35,05 \%)$ fêmeas.

A Organização Mundial da Saúde (2000) estabelece que as populações canina e felina de determinada cidade podem ser estimadas com o emprego da proporção média de cães: humanos de 1:7 (OMS, 2000) e que a de gatos corresponde a $25 \%$ desse valor (ALVES et al., 2005). Considerando-se o último censo demográfico da cidade de Jaboticabal-SP, que estimou uma população de 75.436 pessoas no ano de 2014 (INSTITUTO BRASILEIRO DE GEOGRAFIA E ESTATÍSTICA, 2014), pode-se estabelecer que as populações de cães e de gatos para esse mesmo ano seriam de aproximadamente 10.777 e 2.694 animais, respectivamente. Com esses dados, pode ser inferido que foram castrados em 2014 por volta de $7,12 \%$ e $28,62 \%$ do total da população de cães e gatos, respectivamente. Dessa totalidade, 1.155 eram fêmeas, principalmente caninas, foco para um controle populacional, uma vez que apresentam características fisiológicas consideradas muitas vezes indesejáveis, como o cio, caracterizado por sangramentos e fugas para cópula, e produção de ninhadas indesejáveis aos proprietários (SOTO et al., 2006) que poderão se constituir em animais abandonados, sem controle nas vias públicas. Mas o programa não estabeleceu metas, pois visava principalmente à formação acadêmica 
e profissional dos estudantes e residentes. A conclusão mais adequada sobre os valores permite observar uma evolução do programa ao longo dos anos.

Todo o progresso no programa de castração foi possível devido ao aumento de interesse da comunidade acadêmica em participar como voluntária, mudando a realidade do início do programa, quando contava com a participação de apenas dois residentes e quatro pós-graduandos. Matera (2008) relatou que as aulas teóricas e práticas são o momento para a transmissão dos conhecimentos, atitudes e habilidades, e que isso só é possível com prática em contato direto com a realidade, como a que ocorre em projetos de extensão em que tudo que é ensinado em aula é reunido em diferentes circunstâncias. Aliar essa prática para o desenvolvimento de um programa de cunho social é uma maneira de o aluno construir o seu conhecimento e personalidade profissional. Budziak et al. (2010) e Bürger et al. (2013) também observaram que os programas de esterilização de animais com foco na pesquisa e ensino, além de beneficiarem a saúde pública, proporcionam aos acadêmicos uma aplicação prática dos conhecimentos, o exercício da interdisciplinaridade e os convence da importância da realização e participação em um programa de extensão. A evidência para as afirmações acima fica clara com o relato da acadêmica Amanda Tartarelli: "Sou bolsista no Centro de Esterilização de Cães e Gatos (CECG) desde o primeiro semestre de 2013. Iniciei as atividades como voluntária e, posteriormente, foi-me oferecida uma bolsa por Projeto de Extensão. Desde o início fui encorajada a participar das cirurgias junto aos médicos-veterinários voluntários, que sempre se mostravam dispostos a ensinar. Desta forma, ao longo de três anos, pude acompanhar centenas de procedimentos cirúrgicos como castrações, exérese de tumores, cesáreas, mastectomias, entre outros. Também tive a oportunidade de praticar diversas técnicas utilizadas na rotina clínica, como administração de fármacos, contenção dos animais, realização da assepsia anterior à cirurgia. Esse aprendizado permitiu a complementação de minha formação acadêmica" (TARTARELLI, 2016). Essa mesma aluna também teve a oportunidade de representar o projeto em quatro congressos universitários, apresentando a evolução dele mesmo e os resultados obtidos.

A partir do ano de 2013, o projeto contou com a colaboração rotineira (quatro horas semanais) dos médicos-veterinários residentes (seis alunos) por meio de uma parceria com o Hospital Veterinário "Governador Laudo Natel”, da mesma universidade. Essa participação constante garantiu uma atividade efetiva do projeto ao longo dos anos de 2013 e 2014. Para esses residentes, os benefícios adquiridos dizem respeito à prática cirúrgica. A realização de diferentes técnicas de castração (ovariectomia, ovário-histerectomia e orquiectomia), o conhecimento anatômico e das práticas de instrumentação são os pontos fortes na troca de experiências repassadas pelos veterinários, pesquisadores e professores participantes.

Outro ponto favorável é a ampliação da área de fomento de pesquisas veterinárias e da necessidade de dados e amostras biológicas para o desenvolvimento delas, o que faz do programa uma fonte de coleta de dados e/ou material biológico (sangue, urina, tecido, etc.). Em retribuição, os pesquisadores colaboram como voluntários, possibilitando o aumento do número de animais atendidos, além de orientarem a realização dos procedimentos como citado anteriormente. Durante os anos de 2012 a 2014, quatro projetos de pesquisa foram beneficiados pelo programa de extensão: "Adição de vitamina $\mathrm{E}$ ao meio de vitrificação na viabilidade e desenvolvimento in vitro de oócitos de gatas domésticas pós-descongelação” (FAPESP 2012/01580-8) - Raquel Ribeiro Gutierrez (mestrado); "Uso de antioxidantes no meio diluente Botu-crio ${ }^{\oplus}$ para congelação de células espermáticas provenientes de epidídimo de gatos submetidos à orquiectomia eletiva" (FAPESP 2011/151488) - Beatrice Ingrid Macente (mestrado); "Estudo da maturação in vitro de oócitos caninos em meio seriado de hormônio luteinizante (LH) e progesterona" (FAPESP: 2009/07346-4) - Tathiana Ferguson Motheo (doutorado); "Qualidade das células espermáticas criopreservadas obtidas de tecido testicular de gatos domésticos: influência do tamanho dos fragmentos, avaliação dos crioprotetores e taxa de desenvolvimento embrionário pós ICSI" (Capes) - Beatrice Ingrid Macente (doutorado).

Rotineiramente, o programa é visitado por médicos-veterinários ligados a prefeituras de municípios de todo o Estado de São Paulo interessados em acompanhar os procedimentos realizados, servindo como modelo para aplicação em seus próprios municípios.

Foi perceptível ainda um interesse da sociedade, seja por parte dos proprietários ou pelos voluntários que visam à castração de animais não domiciliados, e em muitos casos, a sua doação. Informações errôneas, como a necessidade de as fêmeas gestarem ou apresentarem o primeiro cio antes da castração e a perda da virilidade dos machos após a retirada dos testículos, são obstáculos que dificultam o aceite da realização da castração, como também foi observado por Silvano et al. (2010), que encontrou uma resistência da população ao ato em questão. Assim como fizeram esses autores, as informações foram devidamente corrigidas pelos participantes do programa durante as orientações transferidas aos proprietários e voluntários.

\section{Conclusões}

- O desenvolvimento de programas de controle esterilização de cães e gatos, desenvolvidos em conjunto com instituições de ensino, aliando pesquisa às práticas, é uma 
opção eficiente e viável a ser explorada por instituições de ensino.

- A participação de estudantes e médicos-veterinários apresenta-se como uma excelente forma de troca de conhecimentos e desenvolvimento das práticas clínica e cirúrgica.

- A associação de projetos sociais ao desenvolvimento de pesquisas favorece o acesso à coleta de dados. (a)

\section{Referências}

ACHA, P. N.; SZYFRES, B. Filariasis zoonóticas. In: ACHA, P. N. Zoonosis y enfermidades transmisibles comunes al hombre y a los animales. 3. ed. Washington: OPS, 2003. v. 3, p. 284-291.

ALMEIDA, E. S. et al. Estudo do destino dos cães no canil municipal de Botucatu antes e após a Lei $\mathrm{n}^{\circ} 12.916$ que dispõe sobre o controle da reprodução de cães. Veterinária e Zootecnia, São Paulo, v. 21, n. 3, p. 433-439, set. 2014.

ALVES, M. C. P. G. et al. Dimensionamento da população de cães e gatos do interior do Estado de São Paulo. Revista de Saúde Pública, São Paulo, v. 39, n. 6, p. 891-897, dez. 2005.

ARMSTRONG, S. J.; BOTZLER, R. G. The animal ethics reader. London: Routledge, 2008 BEAVER, B. V. Comportamento canino: um guia para veterinários. São Paulo: Roca, 2001. BIONDO, A. W. et al. Carrocinha não resolve. Conselho Regional de Medicina Veterinária - PR, Curitiba, ano V, n. 25, p. 20-21, out./dez. 2007.

BUDZIAK, C. et al. A importância do projeto Campanha de Castração na formação do profissional médico-veterinário. Revista Acadêmica Ciência Agrária e Ambiental, Curitiba, v. 8, n. 3, p. 361-370, jul./set. 2010

BÜRGER, K. P. et al. Projeto de esterilização de cães e gatos no município de Descalvado-SP: "Esterilize seu animal: um ato de responsabilidade e amor". Em Extensão, Uberlândia, v. 12 n. 2, p. 93-99, jul./dez. 2013

CATAPAN, D. C. et al. Impacto do programa de esterilização cirúrgica na população de cães e gatos do município de São José dos Pinhais - PR. Revista Brasileira de Ciência Veterinária, Niterói, v. 21, n. 3, p. 178-182, jul./set. 2014

CONCANNON, P. W.; MEYERS-WALLEN, V. N. Currents and proposed methods for contraception and termination of pregnancy in dogs and cats. Journal of the American Veterinary Medical Association, Ithaca, N.Y, v. 198, n. 7, p. 1214-1224, Apr. 1991.

GARCIA, R. C. M.; MALDOMADO, N. A. C.; LOMBARDI, A. Controle Populacional de cães e gatos - aspectos éticos. Ciência Veterinária nos Trópicos, Recife, v. 11, p. 106-110, abr. 2008. Suplemento 1.

GENARO, G. Gato doméstico: futuro desafio para controle da raiva em áreas urbanas? Pesquisa Veterinária Brasileira, Rio de Janeiro, v. 30, n. 2, p. 186-189, fev. 2010.

GOETHEM, B. V.; OKKENS, A. S.; KIRPENSTEIJN, J. Making a rational choice between ovariectomy and ovariohysterectomy in the dog: a discussion of the benefits of either technique. Veterinary Surgery, Philadelphia, v. 35, n. 2, p. 136-143, Feb. 2006

INSTITUTO BRASILEIRO DE GEOGRAFIA E ESTATÍSTICA. Cidades. 2014. Disponível em <http://www.cidades.ibge.gov.br/xtras/perfil.php?lang=\&codmun=352430\&search=saopauloljaboticaballinfograficos:-informacoes-completas>. Acesso em: 7 mar. 2014.

JOFFILY, D. et al. Medidas para o controle de animais errantes desenvolvidas pelo grupo pet medicina veterinária da Universidade Federal Rural do Rio de Janeiro. Em extensão, Uberlândia, v.12, n. 1, p. 197-211, jan./jun. 2013.

MACKAY, C. A. Veterinary practitioners role in pet overpopulation. Journal of the American Veterinary Medical Association, Ithaca, v. 202, n. 6, p. 918-921, Mar. 1993.

MARCHAND, C.; MOORE, A. Pet populations and ownership around the world. Walthan Internacional Focus, London, v. 1, p. 14-15, 1991.

MARTINS, C. M. et al. Mitos e verdades de cães, gatos e seus donos no Brasil. Clínica Veterinária, São Paulo, ano 18, n. 102, p. 32-33, jan./fev. 2013.
MATERA, J. M. O ensino de cirurgia: da teoria à prática. Ciência Veterinária nos Trópicos, Recife, v. 11, p. 96-101, abr. 2008. Suplemento 1.

OFFICE INTERNATIONAL DES EPIZOOTIES. World Organization for Animal Health. Stray dog population control. In: OIE. Terrestrial Animal Health Code. Paris: OIE, 2010. cap. 7.7. Disponível em: <http://web.oie.int/eng/normes/mcode/a_summry.htm>. Acesso em: 28 abr. 2015.

ORGANIZAÇÃO MUNDIAL DA SAÚDE. Guidelines for dog population management Geneva: WHO/WSPA, 2000

ORGANIZAÇÃO PAN-AMERICANA DE SAÚDE. Atenção Primária Ambiental. Brasília, 1999. (Informe Técnico OPS/BRA/HEP/001/1999). Disponível em: <http://www.paho. org/bra/index.php?option=com_content $\&$ view=article \&id=388:atencao-primaria-ambient al\&catid=992: desenvolvimento-sustentavel-submenu>. Acesso em: 12 nov. 2014.

ROMAGNOLI, S. Surgical gonadectomy in the bitch \& queen: should it be done and at what age? In: SOUTHERN EUROPEAN VETERINARY CONFERENCE \& CONGRESO NACIONAL, 2008, Barcelona. Anais... Espanha: SEVC; Espanha: AVEPA, 2008

SANTANA, L. R.; OLIVEIRA, T. P. Guarda responsável e dignidade dos animais. Salvador: Relatório do Ministério Público, 2008.

SÃO PAULO (Estado). Lei n. 12.916, de 16 de abril de 2008. Dispõe sobre o controle da reprodução de cães e gatos e dá providências correlatas. Diário Oficial do Estado de São Paulo, São Paulo, SP, 16 abr. 2008. Seção 1, p. 1-3.

SILVA, M. S.; VASCONCELOS, S. D. Extensão universitária e formação profissional: avaliação da experiência das ciências biológicas na Universidade Federal de Pernambuco. Estudos em Avaliação Educacional, São Paulo, v. 17, n. 33, p. 119-135, jan./abr. 2006.

SILVANO, D. et al. Divulgação dos princípios da guarda responsável: uma vertente possível no trabalho de pesquisa a campo. Revista Eletrônica Novo Enfoque, São Paulo, v. 9, n. 9 , p. 64-86, jan. 2010.

SOTO, F. R. M. et al. Dinâmica populacional canina no Município de Ibiúna - SP: estudo retrospectivo. Brazilian Journal of Veterinary Research and Animal Science, São Paulo, v. 43, n. 2, p. 178-185, 2006.

TARTARELLI, A. Amanda Tartarelli: depoimento. [mar. 2016]. Entrevistadores: MACENTE, B. I. et al. Jaboticabal, 2016.

THRUSFIELD, M. Epidemiologia Veterinária. 2. ed. São Paulo: Roca, 2004

VIEIRA, A. M. L. et al. Programa de controle de populações de cães e gatos do Estado de São Paulo. Boletim Epidemiológico Paulista, v. 6, maio 2009. Suplemento 7. Disponíve em: <http://www.saude.sp.gov.br/resources/ccd/publicacoes/publicacoes-ccd/manuaisnormas-e-documentos-tecnicos/manuaisnormasedocumentostecnicos1_-_manual_de_ controle_de_populacoes_de_caes_e_gatos_no_estado_de_sao_paulo_-_2009.pdf>. Acesso em: 11 nov. 2014. 